\title{
Obtaining the (dust-obscured) star formation history using the VLA-COSMOS survey
}

\author{
V. Smolčić ${ }^{1}$, E. Schinnerer ${ }^{1}$, M. Scodeggio ${ }^{2}$, C. L. Carilli ${ }^{3}$, \\ P. Franzetti ${ }^{2}$ and K. Jahnke ${ }^{1}$ \\ ${ }^{1}$ Max Planck Institut für Astronomie, Königstuhl 17, Heidelberg, D-69117, Germany \\ email: smolcic@mpia.de \\ ${ }^{2}$ IASF Milano-INAF, Via Bassini 15, I-20133, Milan, Italy \\ ${ }^{3}$ National Radio Astronomy Observatory, P.O. Box 0, Socorro, NM 87801-0387
}

\begin{abstract}
Using the multi-wavelength COSMOS survey (Scoville et al. 2006), we develop a method based on purely photometric data to separate the faint VLA-COSMOS radio population into star-forming (SF) galaxies and active-galactic nuclei (AGN). Based on this classification method we select SF galaxies within our sample and present first results on the cosmic (dustobscured) star-formation history based on VLA-COSMOS (Schinnerer et al. 2006) radio data.
\end{abstract}

Radio emission at $1.4 \mathrm{GHz}(20 \mathrm{~cm})$ is dominated by the emission from $\mathrm{SF}$ galaxies and AGN, hence a reliable SF/AGN separation is essential for a robust determination of SF rates utilizing radio data. While AGN dominate radio counts at larger flux densities, their contribution compared to SF galaxies at low fluxes $(<1 \mathrm{mJy})$ is still controversial. The VLA-COSMOS survey $(1 \sigma=10 \mu \mathrm{Jy})$ at $1.4 \mathrm{GHz}$ provides enough targets to allow for a statistically significant characterization of the 'population mix' of faint radio sources: Our sample consists of $\sim 2000$ radio sources that have an optical counterpart within $1.5^{\prime \prime}$. Out of this sample we select $\mathrm{SF}$ galaxies in two steps:

(a) We exclude broad line AGN (type 1) by rejecting objects that show medium or hard X-ray emission and are defined as point sources in HST images (Leauthaud et al. 2006) or visually classified as containing a point-source.

(b) We exclude narrow line AGN (type 2) by fitting the UV to NIR spectral energy distribution of our sources with PEGASE stellar population synthesis models using a package called GOSSIP (Franzetti 2005). We derive the rest-frame P1 color (see Smolčić et al. 2006) and select SF galaxies by applying $P 1 \leqslant 0$. A sample of SF galaxies selected in a such a way is $\sim 65 \%$ complete and up to $\sim 20 \%$ 'contaminated' by AGN type 2 .

Our preliminary results indicate that $\mathrm{SF}$ galaxies are not the dominant radio population at faint flux densities $(<1 \mathrm{mJy})$, but rather have a fairly constant contribution with decreasing fluxes. Without any completeness corrections, we derive lower limits of the SF rate density, $\rho_{*}$, as a function of redshift, following Haarsma et al. (2000) and Condon et al. (1992). $\rho_{*}$ shows, as expected, an increase with redshift up to $z=1$.

\section{References}

Condon, J. J. 1992, ARA\&A, 30, 575

Franzetti, P., 2005, PhD thesis, Universita' di Milano-Bicocca, Milano

Haarsma, D. B., Partridge, R. B., Windhorst, R. A., \& Richards, E. A. 2000, ApJ, 544, 641

Leauthaud, A. et al. 2006, ApJS, in press

Schinnerer, E., Smolčić, V., et al. 2006, ApJS, in press

Scoville, E., et al. 2006, ApJS, in press

Smolčić, V., et al. 2006, MNRAS, 371, 121 